\title{
FETD Study of the Wave Propagation in Chiral Metamaterials
}

\author{
Lei Yang ${ }^{1, *}$, Fuhao Liu $^{2}$ and Wei Yang ${ }^{3}$ \\ ${ }^{1}$ Faculty of Information Technology, Macau University of Science and Technology, Macau, \\ China \\ 2 School of Mathematics and Computational Science, Xiangtan University, Xiangtan, \\ Hunan 411105, China \\ ${ }^{3}$ Hunan Key Laboratory for Computation and Simulation in Science and Engineering \\ and School of Mathematics and Computational Science, Xiangtan University, Xiangtan, \\ Hunan 411105, China
}

Received 30 May 2020; Accepted (in revised version) 27 July 2020

\begin{abstract}
In this paper, we propose a finite element time-domain (FETD) method for the Maxwell's equations in chiral metamaterials (CMMs). The time-domain model equations are constructed by the auxiliary differential equations (ADEs) method. The source excitation method entitled total-field and scattered-field (TF/SF) decomposition technique is applied to FETD method for the first time in simulating the propagation of electromagnetic wave in CMMs, based on which a unified ADE-FETD-UPML$\mathrm{TF} / \mathrm{SF}$ scheme is proposed to simulate the wave in CMMs. The following properties of CMMs can be observed successfully from the numerical experiments based on our method, i.e., the ability of the polarization rotation, and the negative phase velocity. The amplitude of reflected wave can effectively be controlled by the physical parameters of CMMs.
\end{abstract}

AMS subject classifications: 78M10, 35Q61

Key words: Maxwell's equations, finite element time-domain (FETD), chiral metamaterials (CMMs).

\section{Introduction}

As one kind of artificial electromagnetic material, metamaterials are different from the ordinary materials by their special features. One of the most representative features of the metamaterials is their negative refractive index $[9,12]$, which can be obtained when the permittivity and permeability are simultaneously negative [8]. Because of their special

*Corresponding author.

Emails: leiyang@must.edu.mo (L. Yang), liufuhaoedu@163.com (F. H. Liu), yangweixtu@126.com (W. Yang) 
electromagnetic properties, metamaterials have been widely concerned by the scientific community in recent years, and have been successfully applied to the design of radar, antenna, and other microwave devices.

Chirality is a geometric concept, which describes the property of an object or a system that it can be distinguished from its mirror image. There are many examples for the natural chiral materials such as shells, snails, DNA, and for the artificial chiral materials such as spring, spiral ladder. The chiral media [1] are the special materials which can produce the polarization rotation phenomenons. Among those chiral materials, the chiral metamaterial is a new kind of metamaterials with excellent optical activity and circular dichroism, which potentially provides a simpler approach to achieve the negative refraction [2]. The study on CMMs belongs to the category of subwavelength electromagnetics. The chirality can be obviously observed from the electromagnetic wave propagating in the chiral metamaterial. Many amazing electromagnetic characteristics can be generated by the interaction between electromagnetic wave and subwavelength structure, such as negative refractive index, sub-wavelength optical waveguides, circular dichroism, cloaking devices, polarization rotation, etc. [4-7].

Numerical simulation play an important role in the design and application of CMMs. There have been many numerical methods in the market for the purpose, such as the popular finite difference time domain (FDTD) method [1-3,13], method of moments [15] and Transmission Line Matrix (TLM) algorithm [10,14]. As one of the most successful numerical methods, the finite element method has been studied systematically in solving the temporal equations, and has been widely applied in a variety of areas such as computational fluid dynamics, computational electromagnetics and micromagnetics etc.. One outstanding feature is its ability on handling the complex geometry from the practical problems, which also is an attractive feature for the interface problems in the elctromagnetics. In $[11,16,17]$, the finite element time domain (FETD) method has been proposed and explored in the study of electromagnetic (EM) waves.

Although the finite element method has many advantages, as far as we know, there is no work about FETD method which is used to simulate the propagation of electromagnetic wave in CMMs. In this paper, we study the FETD method of the Maxwell's equations for CMMs. Because CMMs belongs to the dispersive media, there have been many methods to deal with the time-domain problems of electromagnetic wave propagation in dispersive media, including auxiliary differential equations (ADEs) method [18], recursive convolution method, shift operator method, and Z-transform method. It is noted that the computational resource required by ADEs method is lower among those methods, so in this paper we use ADE-FETD method to solve the EM problems with both frequency dispersion and chirality at the same time. In general, when the wave source is outside the electromagnetic material, we need to design the incident wave with some special methods. Total-field and scattered-field (TF/SF) decomposition technique has attracted wide attention because of its superior performance on controling the incident error. It is worth to mentioning that TF/SF method has been successfully applied to electromagnetic field simulation in chiral media and metamaterials for FDTD method $[2,13]$. 
Consequently, in this paper, we also employ the TF/SF decomposition technique for the proposed FETD framework in the simulations. Finally, to restrain the reflected wave at the truncated boundary, the UPML method [21] is used.

Based on the above components, a unified framework entitled ADE-FETD-UPML$\mathrm{TF} / \mathrm{SF}$ scheme is proposed for the simulation of electromagnetic (EM) wave propagation in CMMs. Numerical results show the effectiveness of the proposed framework, i.e., from the numerical results the polarization rotation ability of CMMs, and the negative phase velocity can be observed successfully. The amplitude of reflected wave can be effectively controlled by the physical parameters of CMMs.

The paper is organized as follows. In Section 2, time-domain form of the Maxwell's equations are established with ADEs method in dispersive CMMs. In Section 3, the framework ADE-FETD-UMPL-TF/SF is described in detail. In Section 4, numerical results show the singular phenomena (such as cross-polarization, negative refractive index and negative phase velocity [19]) that wave propagation in CMMs slab under the different physical parameters.

\section{Time domain modeling equations in dispersive CMMs and UPML equations}

The constitutive relation for frequency dispersive chiral media can be expressed as follows [2,14]:

$$
\begin{aligned}
& \boldsymbol{D}=\varepsilon(\omega) \boldsymbol{E}-j \kappa(\omega) c^{-1} \boldsymbol{H}, \\
& \boldsymbol{B}=\mu(\omega) \boldsymbol{H}+j \kappa(\omega) c^{-1} \boldsymbol{E},
\end{aligned}
$$

where $c$ is the speed of light in vacuum, $\boldsymbol{D}$ and $\boldsymbol{B}$ are the electric displacement and magnetic induction, respectively, $\varepsilon, \mu$ and $\kappa$, are permittivity, permeability, and chirality parameter [20], respectively

$$
\begin{aligned}
& \varepsilon(\omega)=\varepsilon_{0} \varepsilon_{r}=\varepsilon_{\infty}+\frac{\left(\varepsilon_{s}-\varepsilon_{\infty}\right) \omega_{e}^{2}}{\omega_{e}^{2}-\omega^{2}+2 j \xi_{e} \omega^{\prime}} \\
& \mu(\omega)=\mu_{0} \mu_{r}=\mu_{\infty}+\frac{\left(\mu_{s}-\mu_{\infty}\right) \omega_{h}^{2}}{\omega_{h}^{2}-\omega^{2}+2 j \xi_{h} \omega^{\prime}} \\
& \kappa(\omega)=\frac{\tau_{\kappa} \omega_{\kappa}^{2} \omega}{\omega_{\kappa}^{2}-\omega^{2}+2 j \omega_{\kappa} \xi_{\kappa} \omega^{\prime}}
\end{aligned}
$$

where $\varepsilon_{\infty}, \varepsilon_{s}$ are the permittivities at infinite and zero frequecies; $\mu_{\infty}$ and $\mu_{s}$ are the permeabilities at infinite and zero frequecies; $\xi_{e}, \xi_{h}$ and $\xi_{\kappa}$ are damping factors; $\omega_{e}, \omega_{h}$ and $\omega_{\kappa}$ are resonance angular frequencies; and $\tau_{\kappa}$ is a characteristic time constant describing the magnitude of chirality. 
By the ADEs method, the time-domain governing equations of EM wave in CMMs can be written as follows $[2,14]$ :

$$
\begin{aligned}
& \nabla \times \boldsymbol{H}=\varepsilon_{\infty} \partial_{t} \boldsymbol{E}+\boldsymbol{J}+\boldsymbol{K}_{c}+\boldsymbol{J}_{s}, \\
& \partial_{t^{2}}^{2} \boldsymbol{J}+2 \xi_{e} \partial_{t} \boldsymbol{J}+\omega_{e}^{2} \boldsymbol{J}=\left(\varepsilon_{s}-\varepsilon_{\infty}\right) \omega_{e}^{2} \partial_{t} \boldsymbol{E} \\
& \partial_{t^{2}}^{2} \boldsymbol{K}_{c}+2 \omega_{\kappa} \xi_{\kappa} \partial_{t} \boldsymbol{K}_{c}+\omega_{\kappa}^{2} \boldsymbol{K}_{c}=-\left(\tau_{\kappa} \omega_{\kappa}^{2} / c\right) \partial_{t^{2}}^{2} \boldsymbol{H}, \\
& \nabla \times \boldsymbol{E}=-\mu_{\infty} \partial_{t} \boldsymbol{H}-\boldsymbol{K}-\boldsymbol{J}_{c} \\
& \partial_{t^{2}}^{2} \boldsymbol{K}+2 \xi_{h} \partial_{t} \boldsymbol{K}+\omega_{h}^{2} \boldsymbol{K}=\left(\mu_{s}-\mu_{\infty}\right) \omega_{h}^{2} \partial_{t} \boldsymbol{H} \\
& \partial_{t^{2}}^{2} \boldsymbol{J}_{c}+2 \omega_{\kappa} \xi_{\kappa} \partial_{t} \boldsymbol{J}_{c}+\omega_{\kappa}^{2} \boldsymbol{J}_{c}=\left(\tau_{\kappa} \omega_{\kappa}^{2} / c\right) \partial_{t^{2}}^{2} \boldsymbol{E}
\end{aligned}
$$

where $\partial_{t^{2}}^{2}=\frac{\partial^{2}}{\partial t^{2}}$.

To derive the time-domain uniaxial perfectly matched layer (UPML) model equations used for our chiral metamaterials model, we first rewrite the standard 3D Eqs. (2.3a) and (2.3d) in the frequency domain [21] as follows:

$$
\begin{aligned}
& \nabla \times \boldsymbol{H}=j \omega \varepsilon_{\infty} \boldsymbol{\Lambda} \boldsymbol{E}+\boldsymbol{J}+\boldsymbol{J}_{s}+\boldsymbol{K}_{c}, \\
& \nabla \times \boldsymbol{E}=-j \omega \mu_{\infty} \boldsymbol{\Lambda} \boldsymbol{H}-\boldsymbol{K}-\boldsymbol{J}_{c},
\end{aligned}
$$

where

with

$$
\boldsymbol{\Lambda}=\left[\begin{array}{ccc}
\frac{s_{y} s_{z}}{s_{x}} & 0 & 0 \\
0 & \frac{s_{x} s_{z}}{s_{y}} & 0 \\
0 & 0 & \frac{s_{x} s_{y}}{s_{z}}
\end{array}\right]
$$

$$
s_{x}=\kappa_{x}+\frac{\sigma_{x}}{j \omega \varepsilon_{0}}, \quad s_{y}=\kappa_{y}+\frac{\sigma_{y}}{j \omega \varepsilon_{0}}, \quad s_{z}=\kappa_{z}+\frac{\sigma_{z}}{j \omega \varepsilon_{0}},
$$

where $\kappa_{x}=\kappa_{y}=\kappa_{z}=1, j=\sqrt{-1}$ is the imaginary unit, and $\sigma_{x}, \sigma_{y}$, and $\sigma_{z}$ are the damping functions in the $x-, y-$, and $z$ - directions, respectively.

We can obtain the time-domain form of Eqs. (2.4) by utilizing serveral auxiliary variables. If $\partial_{x}=0$ and $\partial_{y}=0$, then $s_{x}=\kappa_{x}, s_{y}=\kappa_{y}$, the 1D-time-domain forms of Eq. (2.4) can be written as follows:

$$
\begin{aligned}
& -\partial_{z} H_{y}=\varepsilon_{\infty} \partial_{t} E_{x}+\left(\varepsilon_{\infty} / \varepsilon_{0}\right) \sigma_{z} E_{x}+J_{x}+K_{c x}+J_{s x}, \\
& \partial_{z} H_{x}=\varepsilon_{\infty} \partial_{t} E_{y}+\left(\varepsilon_{\infty} / \varepsilon_{0}\right) \sigma_{z} E_{y}+J_{y}+K_{c y}+J_{s y}, \\
& -\partial_{z} E_{y}=-\mu_{\infty} \partial_{t} H_{x}-\left(\mu_{\infty} / \varepsilon_{0}\right) \sigma_{z} H_{x}-K_{x}-J_{c x}, \\
& \partial_{z} E_{x}=-\mu_{\infty} \partial_{t} H_{y}-\left(\mu_{\infty} / \varepsilon_{0}\right) \sigma_{z} H_{y}-K_{y}-J_{c y} .
\end{aligned}
$$

In order to obtain simpler forms, we let

$$
\partial_{t} P_{1 i}=J_{i}, \quad \partial_{t} Q_{1 i}=K_{c i}, \quad \partial_{t} P_{2 i}=K_{i}, \quad \partial_{t} Q_{2 i}=J_{c i},
$$


Eqs. (2.3b), (2.3c), (2.3e), (2.3f) can be rewritten as

$$
\begin{aligned}
& \partial_{t} J_{i}+b_{1} J_{i}+b_{2} P_{1 i}=b_{3} E_{i}, \\
& \partial_{t} K_{c i}+c_{1} K_{c i}+c_{2} Q_{1 i}=c_{3} \partial_{t} H_{i}, \\
& \partial_{t} K_{i}+d_{1} K_{i}+d_{2} P_{2 i}=d_{3} H_{i}, \\
& \partial_{t} J_{c i}+e_{1} J_{c i}+e_{2} Q_{2 i}=e_{3} \partial_{t} E_{i},
\end{aligned}
$$

where the subscript $i$ represents $x$, and $y$, respectively and

$$
\begin{array}{lll}
b_{1}=2 \xi_{e}, & b_{2}=\omega_{e}^{2}, & b_{3}=\left(\varepsilon_{s}-\varepsilon_{\infty}\right) \omega_{e}^{2}, \\
c_{1}=2 \omega_{\kappa} \xi_{\kappa}, & c_{2}=\omega_{\kappa}^{2} & c_{3}=-\tau_{\kappa} \omega_{\kappa}^{2} / c, \\
d_{1}=2 \xi_{h}, & d_{2}=\omega_{h}^{2}, & d_{3}=\left(\mu_{s}-\mu_{\infty}\right) \omega_{h,}^{2} \\
e_{1}=2 \omega_{\kappa} \xi_{\kappa}, & e_{2}=\omega_{\kappa}^{2}, & e_{3}=\tau_{\kappa} \omega_{\kappa}^{2} / c .
\end{array}
$$

\section{The ADE-FETD-UPML-TF/SF scheme for CMMs}

In order to excite electromagnetic wave into the computational region from the point $J_{s}$ in Fig. 1, we use the TF/SF decomposition technique. By the equivalence principle, we can obtain

$$
J_{s}=-n_{\Omega_{t}} \times\left. H_{i}\right|_{\Gamma_{t}},
$$

where

$$
\boldsymbol{n}_{\Omega_{t}}=(0,0,-1)^{T} \quad \text { and } \quad \boldsymbol{J}_{s}=\left[J_{s x}, J_{s y}, J_{s z}\right]^{T}=\left[-H_{i y}, H_{i x}, 0\right]^{T} .
$$

According to TF/SF boundary conditions, we let $J_{s y}=0$. Then the local linear finite element equations in $\Omega^{e}=\left[z_{1}^{e}, z_{2}^{e}\right]$ can be derived from Eqs. (2.5a) and (2.5d) as follows:

$$
\begin{aligned}
& -\sum_{j=1}^{2} H_{y}^{e}(j) A_{i j}^{e}=\sum_{j=1}^{2} \partial_{t} E_{x}^{e}(j) \bar{M}_{i j}^{e}+\sum_{j=1}^{2} E_{x}^{e}(j) \tilde{M}_{i j}^{e}+\sum_{j=1}^{2}\left(J_{x}^{e}(j)+K_{c x}^{e}(j)\right) M_{i j}^{e}+h_{i x}^{e}(i), \\
& \sum_{j=1}^{2} E_{x}^{e}(j) A_{i j}^{e}=-\sum_{j=1}^{2} \partial_{t} H_{y}^{e}(j) \bar{G}_{i j}^{e}-\sum_{j=1}^{2} H_{y}^{e}(j) \tilde{G}_{i j}^{e}-\sum_{j=1}^{2}\left(K_{y}^{e}(j)+J_{c y}^{e}(j)\right) M_{i j}^{e}+h_{y}^{e}(i),
\end{aligned}
$$

where

$$
\left\{\begin{array}{l}
h_{i x}^{e}(i)=\delta_{e, e s}\left(-H_{i y}^{e s}(2)-A_{i, 2}^{e s} H_{i y}^{e s}(2)-\bar{M}_{i, 2}^{e s} \partial_{t} E_{i x}^{e s}(2)\right), \\
h_{y}^{e}(i)=\delta_{e, e s}\left(A_{i, 2}^{e s} E_{i x}^{e s}(2)+\bar{M}_{i, 2}^{e s} \partial_{t} H_{i y}^{e s}(2)\right),
\end{array}\right.
$$

with es is a label of element that represents the first element at the left side of the point $J_{s}$ in Fig. 1, and

$$
\begin{array}{lll}
A_{i j}^{e}=\left(\varphi_{i}^{e}, \partial_{z} \varphi_{j}^{e}\right)_{e}, & \bar{G}_{i j}^{e}=\left(\varphi_{i}^{e}, \mu_{\infty} \varphi_{j}^{e}\right)_{e}, & \tilde{G}_{i j}^{e}=\left(\varphi_{i}^{e}, \frac{\mu_{\infty}}{\varepsilon_{0}} \sigma_{z} \varphi_{j}^{e}\right)_{e}, \\
M_{i j}^{e}=\left(\varphi_{i}^{e}, \varphi_{j}^{e}\right)_{e}, & \bar{M}_{i j}^{e}=\left(\varphi_{i}^{e}, \varepsilon_{\infty} \varphi_{j}^{e}\right)_{e}, & \tilde{M}_{i j}^{e}=\left(\varphi_{i}^{e}, \frac{\varepsilon_{\infty}}{\varepsilon_{0}} \sigma_{z} \varphi_{j}^{e}\right)_{e},
\end{array}
$$




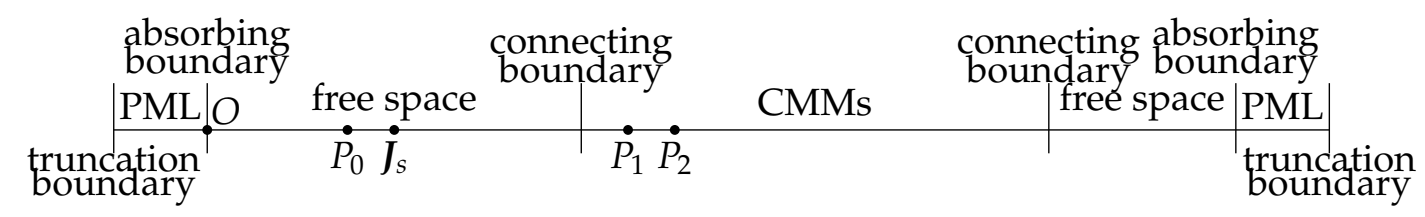

Figure 1: Configuration of 1D ADE-FETD-UPML-TF/SF method for wave propagation into a CMMs slab.

with

$$
(u, v)_{e}=\int_{\Omega^{e}} u(z) \cdot v(z) d z .
$$

We can easily get the semi-discrete global equations as follows:

$$
\begin{aligned}
& -A H_{y}-\bar{M} \partial_{t} E_{x}-\tilde{M} E_{x}-M\left(J_{x}+K_{c x}\right)=h_{i x}, \\
& A E_{x}+\bar{G} \partial_{t} H_{y}+\tilde{G} H_{y}+M\left(K_{y}+J_{c y}\right)=h_{y},
\end{aligned}
$$

where

$$
M_{I J}=\sum_{(i, e)=I(j, e)=J} M_{i j}^{e}
$$

is the global stiffness matrix of $M^{e}$, the global stiffness matrice of $A^{e}, \tilde{M}^{e}, \bar{M}^{e}, \tilde{G}^{e}, \bar{G}^{e}$ can be written similarly, and

$$
h_{i x}(I)=\sum_{(i, e)=I} h_{i x}^{e}(i), \quad h_{y}(I)=\sum_{(i, e)=I} h_{y}^{e}(i) .
$$

Similarly, we can derive the other two semi-descrete global equations from Eqs. (2.5b) and $(2.5 \mathrm{c})$.

Now we can give the fully discrete scheme for the governing Eqs. (2.6), (2.7), (3.5) and the equations derived from (2.5b) and (2.5c). Here we use the leap-log scheme which is an explicit and conditionally stable scheme. In this scheme, the electric field and magnetic current are discrete at the integer time steps, and the magnetic field and electric current are taken at the half-integer time steps. Then the fully discrete equations of 1D ADEFETD-UPML-TF/SF method for CMMs are

$$
\begin{aligned}
& {\left[M_{1}\right]\left\{H^{n+\frac{1}{2}}\right\}=\left[M_{2}\right]\left\{H^{n-\frac{1}{2}}\right\}+[A]\left[\left\{E_{y}^{n}\right\},-\left\{E_{x}^{n}\right\}\right]^{T}-[M]\left(\left\{K^{n}\right\}+\left\{J_{c}^{n}\right\}\right)+\left\{h^{n}\right\},} \\
& \tilde{b}_{+}\left\{J^{n+\frac{1}{2}}\right\}=\tilde{b}_{-}\left\{J^{n-\frac{1}{2}}\right\}-b_{2}\left\{P_{1}^{n}\right\}+b_{3}\left\{E^{n}\right\} \text {, } \\
& \tilde{e}_{+}\left\{K_{c}^{n+\frac{1}{2}}\right\}=\tilde{c}_{-}\left\{K_{c}^{n-\frac{1}{2}}\right\}-c_{2}\left\{Q_{1}^{n}\right\}+\left(c_{3} / \tau\right)\left(\left\{H^{n+\frac{1}{2}}\right\}-\left\{H^{n-\frac{1}{2}}\right\}\right), \\
& {\left[M_{3}\right]\left\{E^{n+1}\right\}=\left[M_{4}\right]\left\{E^{n}\right\}+[A]\left[-\left\{H_{y}^{n+\frac{1}{2}}\right\},\left\{H_{x}^{n+\frac{1}{2}}\right\}\right]^{T}} \\
& -[M]\left(\left\{J^{n+\frac{1}{2}}\right\}+\left\{K_{c}^{n+\frac{1}{2}}\right\}\right)-\left\{h_{i}^{n+\frac{1}{2}}\right\}, \\
& \tilde{d}_{+}\left\{K^{n+1}\right\}=\tilde{d}_{-}\left\{K^{n}\right\}-d_{2}\left\{P_{2}^{n+\frac{1}{2}}\right\}+d_{3}\left\{H^{n+\frac{1}{2}}\right\}, \\
& \tilde{e}_{+}\left\{J_{c}^{n+1}\right\}=\tilde{e}_{-}\left\{J_{c}^{n}\right\}-e_{2}\left\{Q_{2}^{n+\frac{1}{2}}\right\}+\left(e_{3} / \tau\right)\left(\left\{E^{n+1}\right\}-\left\{E^{n}\right\}\right),
\end{aligned}
$$


where

$$
\begin{array}{llll}
\tilde{b}_{+}=\frac{1}{\tau}+\frac{b_{1}}{2}, & \tilde{b}_{-}=\frac{1}{\tau}-\frac{b_{1}}{2}, & \tilde{c}_{+}=\frac{1}{\tau}+\frac{c_{1}}{2}, & \tilde{c}_{-}=\frac{1}{\tau}-\frac{c_{1}}{2}, \\
\tilde{d}_{+}=\frac{1}{\tau}+\frac{d_{1}}{2}, & \tilde{d}_{-}=\frac{1}{\tau}-\frac{d_{1}}{2}, & \tilde{e}_{+}=\frac{1}{\tau}+\frac{e_{1}}{2}, & \tilde{e}_{-}=\frac{1}{\tau}-\frac{e_{1}}{2}, \\
H=\left[H_{x}, H_{y}\right]^{T}, & E=\left[E_{x}, E_{y}\right]^{T}, & J=\left[J_{x}, J_{y}\right]^{T}, & J_{c}=\left[J_{c x}, J_{c y}\right]^{T}, \\
K=\left[K_{x}, K_{y}\right]^{T}, & K_{c}=\left[K_{c x}, K_{c y}\right]^{T}, & h_{i}=\left[h_{i x}, 0\right]^{T}, & h=\left[0, h_{y}\right]^{T}, \\
{\left[M_{1}\right]=\frac{\bar{G}}{\tau}+\frac{\tilde{G}}{2},} & {\left[M_{2}\right]=\frac{\bar{G}}{\tau}-\frac{\tilde{G}}{2},} & {\left[M_{3}\right]=\frac{\bar{M}}{\tau}+\frac{\tilde{M}}{2},} & {\left[M_{4}\right]=\frac{\tilde{M}}{\tau}-\frac{\tilde{M}}{2},}
\end{array}
$$

and

$$
\left[M_{4}\right]\left\{E^{n}\right\}=\left[\left[M_{4}\right]\left\{E_{x}^{n}\right\},\left[M_{4}\right]\left\{E_{x}^{n}\right\}\right]^{T} .
$$

Meanwhile, one can update $\left\{P_{1}\right\},\left\{P_{2}\right\},\left\{Q_{1}\right\},\left\{Q_{2}\right\}$ by the second order central difference quotient formulas of (2.6).

As deduced in Eqs. (3.6), it can be seen that the electric field components and the magnetic field components are $(E x, E y)$ and $\left(H_{x}, H_{y}\right)$, respectively. The electromagnetic field components exist in both free space and CMMs area, but the physical quantities $K_{x}$, $J_{x}, K_{c x}, J_{c x}$ and $K_{y}, J_{y}, K_{c y}, J_{c y}$ only exist in CMMs region (see Fig. 1). So the computational cost and computer memory requirement on solving linear equations of FETD method for CMMs are larger compared with those of conventional FETD method for normal media.

\section{Numerical discussion}

CMMs have many good properties for further development and application prospects, such as optical rotation and negative refractive index. However, the manufacture of CMMs can be so complicated that numerical simulations by combining mathematical methods and computers are very essential for the manufacture. In the introduction, we pointed out that when adding sources outside the simulated medium, special sources are usually used to add sources. Due to the high-dimensional situation, this method of adding sources is more complicated. In addition, the calculation of the algorithm increases exponentially with the increase of the dimensions. In this paper, we only consider the $1 \mathrm{D}$ case for some phenomena of CMMs in our simulations.

In the numerical experiments in this section, we use the positive direction of the $z$ axis as the transmission direction of the wave, and take the multiple cycle m-n-m pulse wave (defined in [2]) as the incident wave. The position of the source of the incident wave is $J_{s}$ (see Fig. 1). For the simulated environment (as shown in Fig. 1), we have the following settings: The thickness of the CMMs slab is $2 \lambda_{0}$, and the thickness of the free space on the left of the CMMs is $\lambda_{0}$, on the right, the thickness of the free space is $\lambda_{0} / 2$. The thickness of the PML on both sides is 50 cells, the grid of the spatial layer is taken as $\Delta z=\lambda_{0} / 500$, and the time step is $\Delta t=\Delta z / 3 c$, where $\lambda_{0}=c / f_{0}$ is the wavelength of the incident wave. In 
order to facilitate the observation of physical phenomena, we set the observation points $P_{0}, P_{1}$ and $P_{2}$, and their coordinates are $90 \Delta z, 510 \Delta z$ and $520 \Delta z$, respectively.

\subsection{Case 1: $\left|\varepsilon_{r} \mu_{r}\right|>|\kappa|^{2}$}

The numerical electric and magnetic fields obtained by our ADE-FETD-UPML-TF/SF method at different working frequencies in the dispersive and lossy CMMs slab with $\left|\varepsilon_{r} \mu_{r}\right|>|\kappa|^{2}$ are shown in Fig. 2. The physical material parameters for the CMMs slab are chosen to be $\varepsilon_{s}=4.6 \varepsilon_{0}, \varepsilon_{\infty}=3.6 \varepsilon_{0}, \mu_{s}=1.1 \mu_{0}, \mu_{\infty}=\mu_{0}, \xi_{e}=0.048 \omega_{e}, \xi_{h}=0.009 \omega_{h}$, $\omega_{h}=2 \pi \times 8.5 \mathrm{GHz}, \omega_{e}=\omega_{\kappa}=2 \pi \times 8.25 \mathrm{GHz}, \xi_{\kappa}=0.09$ and $\tau_{\kappa}=1 p s$. In Figs. 2(a)-2(d), the chirality parameter $\kappa \approx-0.0256-0.0023 j$, and the real parts of $\varepsilon_{r} \approx 3.3954-0.0098 j$ and $\mu_{r} \approx 0.9780-0.0002 j$ are both positive at $f_{0}=20 \mathrm{GHz}\left(\lambda_{0} \approx 0.015 \mathrm{~m}\right)$. In Figs. $2(\mathrm{e})-2(\mathrm{~h})$, the chirality parameter $\kappa \approx-0.1096-0.2374 j$, and the real parts of $\varepsilon_{r} \approx-1.3450-5.7111 j$ and $\mu_{r} \approx-1.6538-2.0421 i$ are both negative at $f_{0}=8.6 \mathrm{GHz}\left(\lambda_{0} \approx 0.0349 \mathrm{~m}\right)$. In Figs. $2(\mathrm{~b})$ and 2(f), we can see the dispersive effect in the CMMs as the time step increases.

The leading portion of copolarized $E_{x}$ at $P_{0}$ and cross-polarized $E_{y}, H_{x}$ at $P_{1}, P_{2}$ are shown in Figs. 2(a), 2(e), 2(c)-2(d) and Figs. 2(g)-2(h). The presence of the chiral parameter $\kappa$ causes cross-polarization in CMMs and generates electromagnetic field components $E_{y}$ and $H_{x}$. When the physical parameters of the chiral media are the same, the chiral parameters corresponding to waves with different frequencies are different. Under this premise, the larger the module of $\kappa$ is, the greater the amplitudes of the cross-polarized waves are. In Figs. 2(b) and 2(f), it can be clearly seen that the electric field component $E_{x}$ reaches its peak at the observation points $P_{1}$ and $P_{2}$. When the frequency of the incident wave is $20 \mathrm{GHz}$, the real parts of $\varepsilon_{r}$ and $\mu_{r}$ are both positive, so the peak of $E_{x}$ at $P_{1}$ appears earlier than that at $P_{2}$; However, when the frequency of the incident wave is $8.6 \mathrm{GHz}$, the real parts of $\varepsilon_{r}$ and $\mu_{r}$ are both negative, so the peak of $E_{x}$ at $P_{1}$ appears later than that at $P_{2}$. This is because the latter $\varepsilon_{r}$ and $\mu_{r}$ cause CMMs to have negative refractive index, which causes negative phase velocity in the wave propagation process (see Fig. 2(f)).

\subsection{Case 2: $\left|\varepsilon_{r} \mu_{r}\right|<|\kappa|^{2}$}

In this case, the chirality parameter of the CMMs slab satisfies the conditions $\left|\varepsilon_{r} \mu_{r}\right|<|\kappa|^{2}$. The material parameters of the CMMs slab are chosen as $\varepsilon_{s}=4.4 \varepsilon_{0}, \varepsilon_{\infty}=3.5 \varepsilon_{0}, \mu_{s}=1.1 \mu_{0}$, $\mu_{\infty}=\mu_{0}, \xi_{e}=0.045 \omega_{e}, \xi_{h}=0.08 \omega_{h}, \xi_{k}=0.01, \tau_{k}=1.2 p s, \omega_{e}=2 \pi \times 7.8 \mathrm{GHz}, \omega_{k}=2 \pi \times 8.25 \mathrm{GHz}$ and $\omega_{h}=2 \pi \times 8.2 \mathrm{GHz}$. In Figs. 3(a) and 3(c), the chirality parameter $\kappa \approx-0.5653-0.1064 j$, and the real parts of $\varepsilon_{r} \approx 0.3462-1.2971 j$ and $\mu_{r} \approx 0.2186-0.1056 j$ are both positive at $f_{0}=$ $8.7 \mathrm{GHz}\left(\lambda_{0} \approx 0.0345 \mathrm{~m}\right)$. In Figs. $3(\mathrm{~b})$ and $3(\mathrm{~d})$, the chirality parameter $\kappa \approx-0.9366-0.3137 j$, and the real parts of $\varepsilon_{r} \approx-0.2683-1.9707 j$ and $\mu_{r} \approx-0.2788-0.2846 j$ are both negative at $f_{0}=8.5 \mathrm{GHz}\left(\lambda_{0} \approx 0.0353 \mathrm{~m}\right)$.

Because of the negative refractive index of CMMs, we can clearly see the negative phase velocity in Fig. 3(d).

In Figs. 2(a), 2(e), Figs. 3(a) and 3(b), we can see clearly the reflected waves because of 


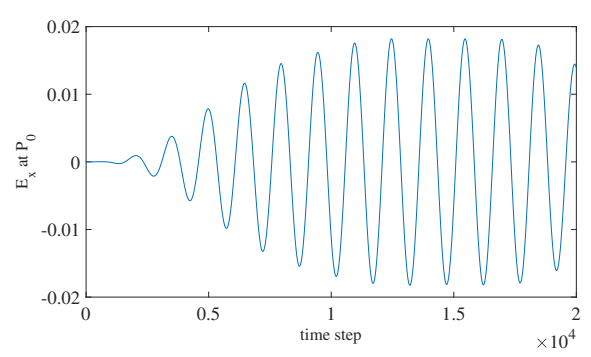

(a)

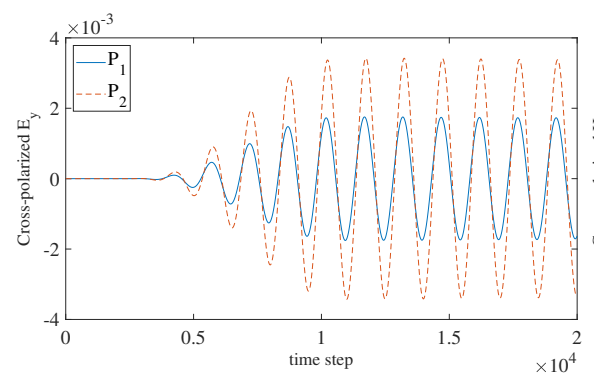

(c)

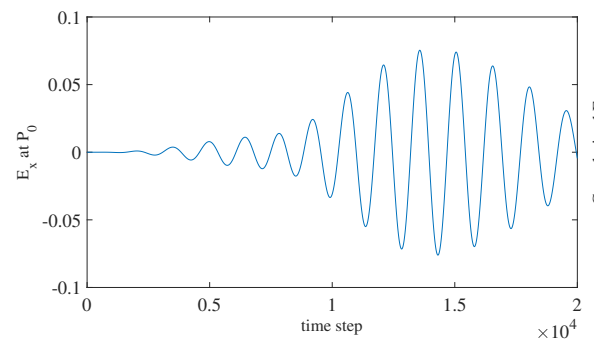

(e)

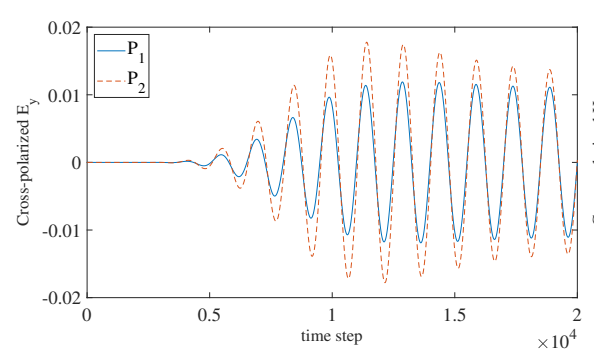

(g)

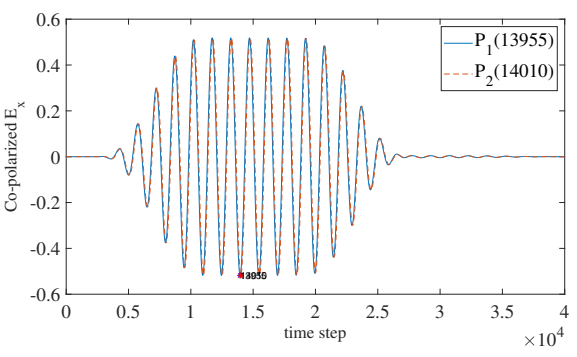

(b)

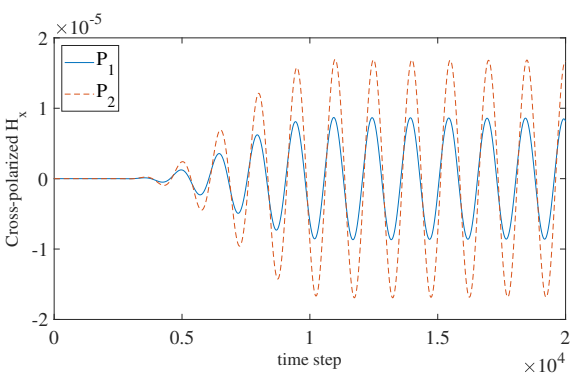

(d)

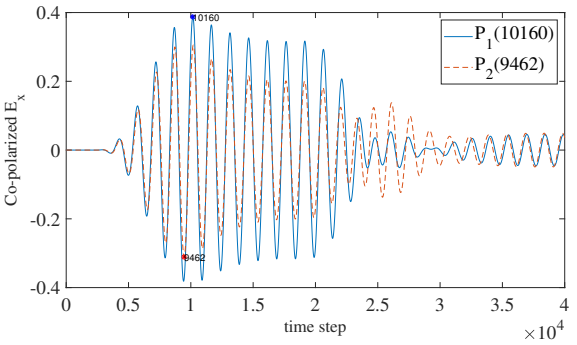

(f)

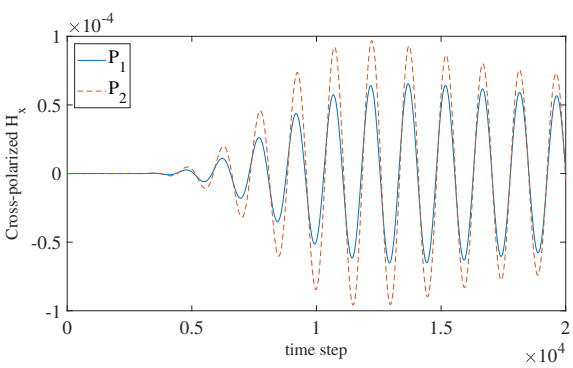

(h)

Figure 2: The obtained copolarized $E_{x}$ and cross-polarized $E_{y}, H_{x}$ in the CMMs slab at the observation points $P_{0}, P_{1}$ and $P_{2}$. (b) and (f): the numbers in the legends are the time steps at which the waves reach their peaks at $P_{1}$ and $P_{2}$, respectively. 


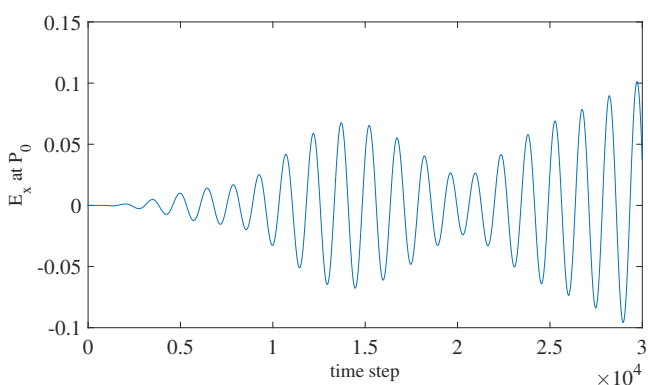

(a)

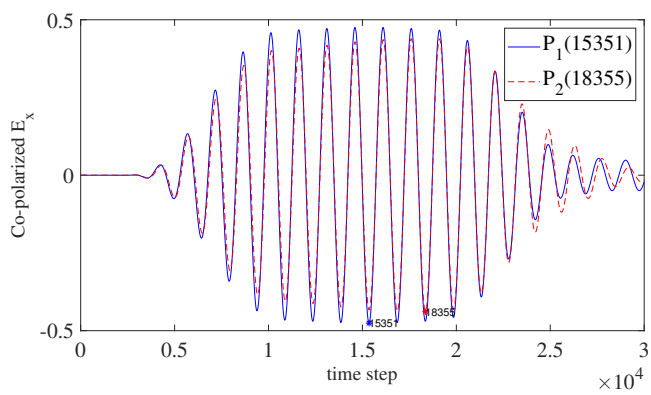

(c)

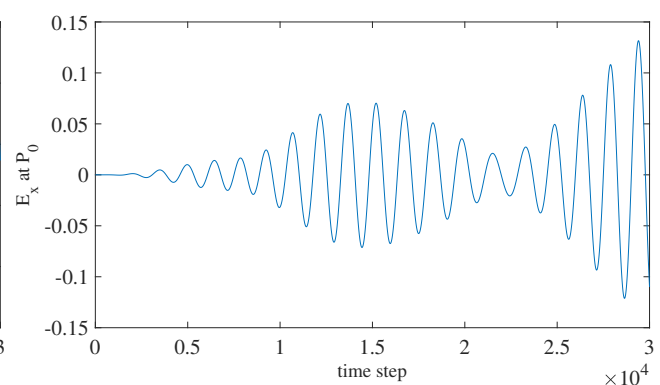

(b)

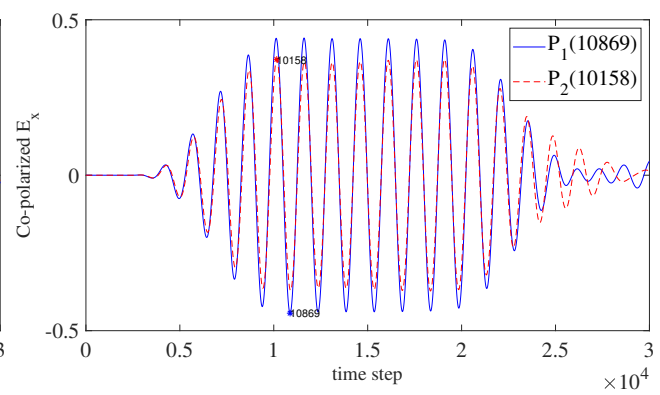

(d)

Figure 3: The obtained copolarized $E_{x}$ in the chiral media and CMMs slab measured at $P_{0}, P_{1}$, and $P_{2}$. (c)-(d): the numbers in the legends are the time steps at which the waves reach their peaks at $P_{1}$ and $P_{2}$.

the mismatch of the impedance matching condition at the interface between the CMMs and free space. In Fig. 2, we see that the amplitudes of the cross-polarized electric field $E_{y}$ and magnetic field $H_{x}$ are satisfied with $A_{E_{y}} / A_{H_{x}} \approx \sqrt{\mu_{\infty} / \varepsilon_{\infty}}$ at the same point. Comparing our numerical results in Figs. 2 and 3, we can conclude that the sign of phase velocity of the wave in the CMMs slab is determined only by the signs of permittivity and permeability, which is the same as the results by using the FDTD method in the paper [2]. And from Figs. 2(a) and 2(e), we can obtain that the amplitude of the reflected wave is related to the chirality parameter $\kappa$.

\section{Conclusions}

In this paper, a unified ADE-FETD-UPML-TF/SF scheme is proposed for the Maxwell's equations in CMMs. In our numerical simulations, the EM wave propagation in $1 \mathrm{D}$ CMMs slab is studied by using the proposed scheme. Numerical results are presented to confirm the effectiveness and applications of our scheme to model many interesting phenomena while wave propagating in the CMMs. In the future, we will design and analyze the FETD method for the three-dimensional Maxwell's equations in chiral media 
and CMMs.

\section{Acknowledgements}

This work was supported partially by the Science and Technology Development Fund, Macau SAR (0070/2019/A2) and National Natural Science Foundation of China, Grant No. 11701598, Scientific Research Fund of Hunan Provincial Science and Technology Department (No. 2018WK4006), NSFC Projects No. 11771371, and Key Project of Hunan Education Department No. 18A056.

\section{References}

[1] R. Mohammadi, M. Dehmollaian, and J. Rashed-Mohassel, A novel FDTD formulation to model dispersive chiral media, 11th European Conference on Antennas and Propagation (EUCAP), (2017), pp. 525-528.

[2] M. Y. WANG, G. P. Li, M. ZhOU, R. WAng, C. L. ZhONG, J. Xu AND H. ZHeng, The effect of media parameters on wave propagation in a chiral metamaterials slab using FDTD, Int. J. Numer. Model. Electronic Networks, Devices Fields, 27(1) (2014), pp. 109-121.

[3] Liping GaO, XiAOrui SANG AND RENGANG SHI, Energy identities and stability analysis of the Yee scheme for 3D Maxwell equations, Numer. Math. Theory Methods Appl., 13(3) (2020), pp. 788-813.

[4] Z. WONG, Y. WANG, K. J O’Brien, J. Rho, X. Yin, S. Zhang, N. X. FANG, T. J. Yen AND $X$. ZHANG, Optical and acoustic metamaterials: superlens, negative refractive index and invisibility cloak, J. Optics, 19(8) (2017), 084007.

[5] H. LIU, Virtual reshaping and invisibility in obstacle scattering, Inverse Problems, 25(4) (2008), 045006.

[6] G. BAO, H. LIU AND J. ZOU, Nearly cloaking the full Maxwell equations: cloaking active contents with general conducting layers, Journal de Mathematiques Pures et Appliquees, 101(5) (2014), pp. 716-733.

[7] Y. DenG, H. LiU AND G. UHLMANN, Full and partial cloaking in electromagnetic scattering, Arch. Rational Mech. Anal., 223(1) (2017), pp. 265-299.

[8] S. ZHANG, Y. S. PARK, J. LI, X. LU, W. ZHANG AND X. ZHANG, Negative refractive index in chiral metamaterials, Phys. Rev. Lett., 102 (2009), 023901.

[9] N. KainA, F. LEMOULT, M. FINK AND G. LeROSEY, Negative refractive index and acoustic superlens from multiple scattering in single negative metamaterials, Nature, 525 (2015), 77.

[10] Rahman Anisur, Wenqian Xu, Kunhang Li, Hua-Shu Dou, Boo Cheong KhOO AND JIE MAO, Influence of magnetic force on the flow stability in a rectangular duct, Adv. Appl. Math. Mech., 11(1) (2019), pp. 24-37.

[11] LEI YANG AND GUANGHUI HU, An adaptive finite element solver for demagnetization field calculation, Adv. Appl. Math. Mech., 11(5) (2019), pp. 1048-1063.

[12] W. Li, Q. MenG, R. HuAng, Z. ZHONG AND B. ZHANG, Thermally tunable broadband terahertz metamaterials with negative refractive index, Optics Commun., 412 (2018), pp. 85-89.

[13] W. YANG, L. LIU AND Y. HUANG, The FDTD simulation for the performance of dispersive cloak devices, Appl. Math. Lett., 88 (2019), pp. 171-178. 
[14] K. Mounirh, S. El Adraoui, Y. Ekdiha, M. Yaich And M. Khalladi, Modeling of dispersive chiral media using the ADE-TLM method, Prog. Electromagnetics Res., 64 (2018), pp. 157-166.

[15] M. S. KLUSKENS AND E. H. NEWMAN, Method of moments analysis of scattering by chiral media, Nasa Sti/recon Technical Report, 187 (1991).

[16] W. YANG, J. LI, AND Y. HUANG, Modeling and analysis of the optical black hole in metamaterials by the finite element time-domain method, Comput. Method. Appl., 304 (2016), pp. 501-520.

[17] W. YANG, J. Li AND Y. HUANG, Mathematical analysis and finite element time domain simulations of arbitrary star-shaped ectromagnetic cloaks, SIAM J. Numer. Anal., 56(1) (2018), pp. 136-159.

[18] M. FujiI, M. TAhARA, I. SAKAgAmi, W. FreUde, AND P. Russe, High-order FDTD and auxiliary differential equation formulation of optical pulse propagation in 2-D Kerr and Raman nonlinear dispersive media, IEEE J. Quantum Electronics, 40(2) (2004), pp. 175-182.

[19] T. G. MACKAY AND A. LAKHTAKIA, Plane waves with negative phase velocity in faraday chiral mediums, Phys. Rev. E, 69 (2004), 026602.

[20] A. H. SihvOLA, Electromagnetic modeling of bi-isotropic media, Prog. Electromagnetic Res., 9 (1994), pp. 45-86.

[21] J. Li AND Y. HUANG, Time-Domain Finite Element Methods for Maxwell's Equations in Metamaterials, Springer Berlin Heidelberg, Berlin, Heidelberg, (2013). 